\title{
Amazônia Oriental, sistemas alternativos ao de corte e queima utilizados na agricultura de subsistência
}

\section{Eastern Amazonia, alternative systems to cutting and burning used in subsistence agriculture}

\author{
Eleneide Doff Sotta ${ }^{1}$, Robson Carmo Lima $2^{2 *}$, Mikael Bruno Brito Ramos $0^{3}$, Breno \\ Marques da Silva e Silva 4 , Perseu da Silva Aparício 5
}

${ }^{1}$ Engenheira Florestal, Doutorado em Forestry and Forest Ecology pelo Universitat Goettingen, Pesquisadora cedida do Ministério da Agricultura, Pecuária e Abastecimento, Brasil. E-mail: esotta@gmail.com

${ }^{2}$ Engenheiro Florestal, Mestre em Ciências Florestais pela Universidade Federal Rural de Pernambuco, Doutorando em Biodiversidade e Biotecnologia pela Rede Bionorte pela Universidade Federal do Estado do Amapá. Brasil. E-mail: robsoncl91@hotmail.com * Autor para correspondência

${ }^{3}$ Acadêmico de Engenharia Florestal do Instituto Macapaense de Ensino Superior-IMMES Brasil. E-mail: mkl.brito12@gmail.com ${ }^{4}$ Biólogo, Doutor em Agronomia pela UNESP, Docente da Universidade do Estado do Amapá-UEAP. Brasil. E-mail: silvabms@hotmail.com ${ }^{5}$ Engenheiro Florestal, Doutor em Biodiversidade Tropical pela Universidade Federal do Amapá, Docente da Universidade do Estado do AmapáUEAP. Brasil.E-mail: perseu_aparicio@yahoo.com.br

\section{Palavras-chave \\ Práticas de manejo \\ Agricultura \\ Desmatamento}

\section{Keywords}

Management practices Agriculture

Deforestation

\begin{abstract}
A agricultura itinerante ou agricultura de corte e queima, apresenta uma área de cultivo, onde o corte da vegetação é feito no período seco e a queima no início da estação chuvosa. Essas práticas envolvem o desmatamento e a queima da vegetação para implantar a agricultura itinerante na região amazônica, tendo como consequência o desgaste do solo e a emissão de gases prejudiciais ao meio ambiente. $O$ uso de práticas de manejo do solo que promovam maior incorporação do carbono atmosférico e aumentam a produtividade (em tempo e qualidade), reduzindo o uso de novas áreas de floresta para agricultura são essenciais. Logo, o objetivo deste estudo é determinar o sistema de manejo de solo que propicie maior fixação de carbono (C) em solos utilizados para a agricultura de subsistência na Amazônia. $O$ estudo foi realizado no município de Macapá/AP em uma propriedade particular no distrito São Joaquim do Pacuí $\left(0^{\circ} 48^{\prime} 38^{\prime \prime} \mathrm{N}\right.$ e $\left.50^{\circ} 45^{\prime} 59^{\prime \prime} \mathrm{O}\right)$. A vegetação da área é caracterizada como floresta de transição entre Cerrado e Floresta Ombrófila Densa, com espécies de 8 a $12 \mathrm{~m}$ de altura. As amostragens foram realizadas nos seguintes tratamentos: capoeira triturada $(T)$, capoeira queimada primeiro ciclo de cultivo (Q1), capoeira queimada segundo ciclo cultivo (Q2) e o controle com capoeira (C). Os resultados obtidos para os tratamentos mostraram os sistemas de manejo com trituração da capoeira (T) e queima da capoeira no primeiro ciclo de cultura (Q1) indicaram maior incorporação de carbono total no solo.
\end{abstract}

The itinerant agriculture or agriculture of cut and burn, presents an area of cultivation, where the cut of the vegetation is made in the dry period and the burning in the beginning of the rainy season. These practices involve the deforestation and burning of vegetation to implement itinerant agriculture in the Amazon region, resulting in soil wear and the emission of harmful gases to the environment. The use of soil management practices that promote greater incorporation of atmospheric carbon and increase productivity (in time and quality), reducing the use of new forest areas for agriculture are essential. Therefore, the objective of this study is to determine the soil management system that provides greater carbon fixation (C) in soils used for subsistence agriculture in the Amazon. The study was conducted in the municipality of Macapá/AP on a private property in the district of São Joaquim do Pacui $\left(0^{\circ} 48^{\prime} 38^{\prime \prime} \mathrm{N}\right.$ and $\left.50^{\circ} 45^{\prime} 59^{\prime \prime} \mathrm{O}\right)$. The vegetation of the area is characterized as a transition forest between Cerrado and Dense Ombrophylous Forest, with species from 8 to $12 \mathrm{~m}$ in height. Sampling was performed in the following treatments: crushed capoeira (T), burnt capoeira first cultivation cycle (Q1), burnt capoeira second cultivation cycle (Q2) and control with capoeira (C). The results obtained for the treatments showed the management systems with crushing of capoeira $(T)$ and burning of capoeira in the first culture cycle (Q1) indicated greater incorporation of total carbon in the soil.

\section{INTRODUÇÃO}

As mudanças climáticas globais são intensamente influenciadas por gases de efeito estufa, principalmente dióxido de carbono ( $\mathrm{CO} 2)$, metano $(\mathrm{CH} 4)$ e óxido nitroso (N2O). Esses gases são emitidos de forma acentuada, a partir 
das práticas amplamente utilizadas no preparo das áreas destinadas à pecuária e a agricultura (BERVALD, 2005). Essas práticas envolvem o desmatamento e a queima da vegetação para implantar a agricultura itinerante na região amazônica (FEARNSIDE 1996; 2007).

A agricultura itinerante ou agricultura de corte e queima, apresenta uma área de cultivo geralmente de um a três hectares, com clima favorável, solos planos manejáveis e próximos às residências. Nesse tipo de cultivo o corte da vegetação é feito no período seco e a queima no início da estação chuvosa. Em seguida é feito o plantio de espécies agrícolas para produção e consumo familiar (JÚNIOR, MURRIETA e ADAMS, 2008).

Com dois a três anos de uso, as áreas de cultivo apresentam diminuição da taxa de nutrientes disponível no solo. Alguns dos fatores para essa diminuição é a volatilização dos nutrientes pela queima e oxidação da matéria orgânica, além do que o solo sem vegetação fica susceptível à erosão hídrica e à lixiviação dos minerais presentes nas cinzas resultantes da queima. Esses fatores somados a demanda nutricional do cultivo, podem resultar na deterioração física do solo e na consequente redução da produção agrícola (LAMPRECHT, 1990), o que impõe a um período de pousio, sendo essa técnica utilizada para preservar a terra que mantém uma área sem cultivo por certo período para restabelecer os nutrientes perdidos com o plantío anterior. É um período em que a terra fica em repouso, isto é, uma área é mantida sem lavoura alguma por um espaço de tempo (CIRNE, 2014).

Entretanto, o sistema de corte e queima usado como forma de preparo do solo pode ser um sistema sustentável na agricultura. A queimada vegetação disponibiliza nutrientes para a fertilização do solo, aumenta o pH e reduz a quantidade de ervas daninhas no cultivo agrícola em longo prazo (KATO; DENICH; VLEK, 1999). Porém, essa queima emite gases de efeito estufa e induz a uma grande perda das propriedades físicas e químicas do solo.

$\mathrm{Na}$ busca por sistemas alternativos ao de corte e queima que possam ser aplicados no manejo do solo para a implantação de cultivos agrícolas, diversas opções são propostas aos agricultores da Amazônia brasileira. Dentre os sistemas de cultivo, o preparo convencional e o plantio direto são os que apontam menor agregação no preparo convencional do solo (CARPENEDO E MIELNICZIUK, 1990). Outros sistemas como o corte e trituração da capoeira foram testados na agricultura visando à geração de tecnologias de manejo do solo que assegurem uma produção agrícola sustentável na região.

Neste contexto, é que o presente estudo tem como objetivo determinar o sistema de manejo de solo que propicia uma maior incorporação de carbono (C) em solos utilizados para a agricultura de subsistência na Amazônia.

\section{MATERIAL E MÉTODOS}

\section{Área de estudo}

O estudo foi realizado em uma fazenda no distrito São Joaquim do Pacuí, zona rural do município de Macapá/AP, $\left(0^{\circ} 48^{\prime} 38^{\prime \prime} \mathrm{N}\right.$ e $\left.50^{\circ} 45^{\prime} 59^{\prime \prime} \mathrm{O}\right)$ (Figura 1$)$.

Figura 1. Localização da área de estudo, propriedade particular em São Joaquim do Pacuí, no estado do Amapá

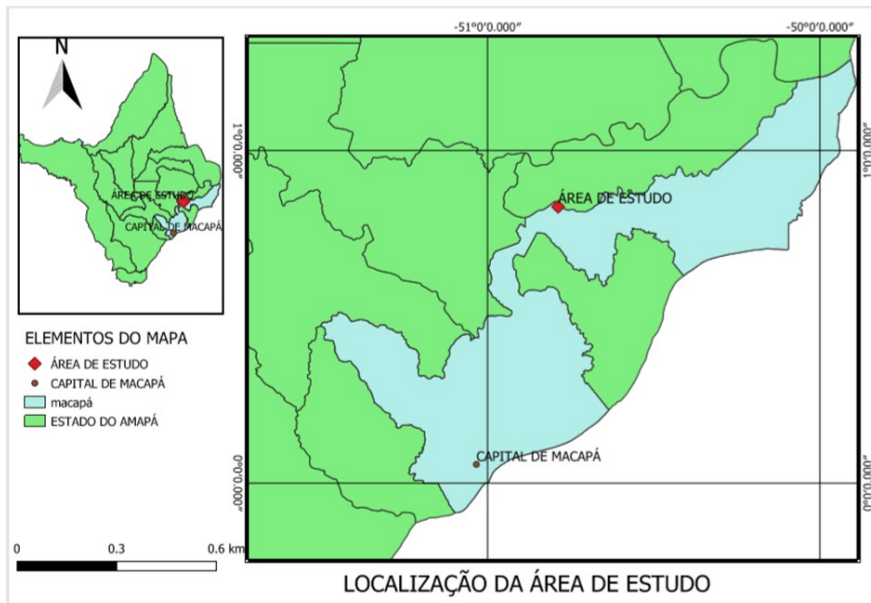

O clima da área de estudo, segundo Koppen é classificado como Ami-Tropical Chuvoso com índice pluviométrico anual médio de $2700 \mathrm{~mm}$, com temperatura média anual de 26 ㄷ. O período chuvoso ocorre entre janeiro e junho, sendo o mês de março com pico mais alto de chuva. E período de estiagem, com pico mais seco no mês de setembro (SILVA, 2009).

A vegetação da área é caracterizada como floresta de transição entre Cerrado e Floresta Ombrófila Densa (IEPA, 2003), com espécies de 8 a $12 \mathrm{~m}$ de altura, sendo alguns componentes dessa vegetação: o morototó (Araliaceae: Shefflera morototoni Aubl.); muiratinga (Moraceae: Maquira sclerophylla Ducke); pitaíca (Fabaceae: Swartzia polyphylla DC.); pracaxí (Fabaceae: Pentaclethra macroloba Benth.); e visgueiro (Fabaceae: Parkia pendula Willd.).

O solo da área de estudo é arenoso, apresentando baixa fertilidade natural, baixo teor de matéria orgânica, média acidez e textura média, com predominância de Latossolo Amarelo distrófico, o qual tem condição favorável ao estabelecimento de práticas agronômicas (IEPA, 2002) (tabela $1)$. 
Tabela 1. Análise dos atributos químicos e físicos do solo da unidade experimental do PACUÍ, Amapá.

\begin{tabular}{|c|c|c|c|c|c|c|c|c|c|c|c|c|c|c|}
\hline Área & $\mathrm{pH}$ & M. 0. & $\mathbf{P}$ & $\mathrm{K}^{+}$ & $\mathrm{Ca}^{2+}+\mathrm{Mg}^{2+}$ & $\mathrm{Ca}^{2+}$ & $\mathrm{Al}^{3+}$ & $\mathrm{H}^{+}+\mathrm{Al}^{3+}$ & $S$ & СтC & $\mathbf{v}$ & m & Argila & Areia \\
\hline & $\mathrm{H}_{2} \mathrm{O}$ & $\mathrm{g} \mathrm{kg}^{-1}$ & $\mathrm{mgdm}^{-3}$ & & ----------.-- & $----C_{r}$ & $10 I_{c} d m$ & 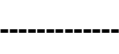 & ----- & --- & $\%$ & $\%$ & $\mathbf{g ~ k g}^{-1}$ & $\mathbf{g ~ k g}^{-1}$ \\
\hline PACUÍ & 5,0 & 41 & 12 & 0,06 & 0,4 & - & 0,7 & 10,73 & 0,5 & 11,23 & 4 & 58 & 259 & 566 \\
\hline
\end{tabular}

\section{Sistema de amostragem e coleta de amostras de solo}

A amostragem, com coleta de material foi realizada em junho de 2009, na área de estudo, dois anos após a implantação dos experimentos pelo projeto TIPITAMBA.

A unidade amostral da propriedade em São Joaquim do Pacuí abrange uma área de aproximadamente 2,0 ha, onde foram divididas por quatro parcelas de 0,5 ha, sendo uma destas uma área de capoeira de 13 anos, que foi amostrada como área testemunha. 0 experimento seguiu o método de amostragem sistemática (Figura 2). As amostragens foram realizadas nos seguintes tratamentos: capoeira triturada (T), capoeira queimada primeiro ciclo de cultivo (Q1), capoeira queimada segundo ciclo cultivo (Q2) e o controle com capoeira (C).

Figura 2. Esquema representativo da disposição das parcelas e das amostras compostas dentro de cada parcela, em São Joaquim do Pacuí, Amapá.

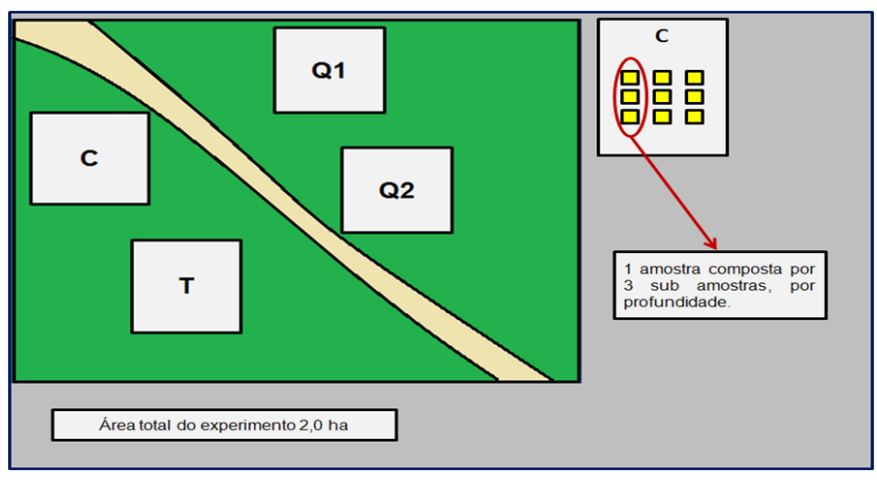

Para a trituração da capoeira foi utilizado um frezador florestal, equipamento denominado Tritucap, o qual foi acoplado a um trator $4 \times 4$ com super redução especialmente adaptado para realizar a operação de trituração da biomassa. A quantidade de biomassa seca resultante da trituração feita pelo frezador foi de 69,4 tha-1.

Nos tratamentos com capoeira queimada Q1 e Q2 o preparo do solo foi feito como tradicionalmente é realizado pelos agricultores, com o corte e queima da capoeira.

Em cada parcela foram coletadas, com auxílio de um trado de análise indeformada, amostras indeformadas de $10 \times 10 \mathrm{~cm}$ de solo nas profundidades de 0 a $5 \mathrm{~cm}, 5$ a $10 \mathrm{~cm}$ e de 10 a 20 cm. A cada profundidade foram coletadas três sub amostras para formar uma amostra composta, totalizando três amostras compostas por tratamento. As amostras foram envolvidas em papel alumínio, colocadas em sacos plásticos e cuidadosamente armazenadas para transporte até o laboratório.

\section{Fracionamento físico granulométrico e densiométrico}

Para a quantificação e qualificação da matéria orgânica no solo foram utilizadas técnicas combinadas de fracionamento granulométrico e densiométrico visando à separação das diferentes classes de agregados do solo e quantificação da matéria orgânica livre e oclusa associada a estes agregados.

No fracionamento granulométrico as amostras foram secas à sombra e separadas em classes de agregados $<0,25$ $\mathrm{mm}$, de 0,25 a $0,5 \mathrm{~mm}$, de 0,5 a $1 \mathrm{~mm}$, de 1 a $2 \mathrm{~mm}$ e $>4 \mathrm{~mm}$, utilizando uma série de peneiras de ferro.

No fracionamento densiométrico foram utilizadas amostras de $20 \mathrm{~g}$ de solo compostas por suas respectivas proporções dentro de cada classe de agregado. Nesse procedimento, as amostras de $20 \mathrm{~g}$ de solo foram colocadas em tubos de ensaio e adicionados $60 \mathrm{ml}$ de politungstato $(1,6$ Mg m-3). Essa mistura foi lentamente agitada e, em seguida, centrifugada por 15 minutos a $2500 \mathrm{rpm}$. Após a centrifugação, retirou-se o sobrenadante por filtragem (sucção), utilizando-se bomba de vácuo, e o filtro usado para reter a matéria orgânica livre foi retirado para secagem.

Em seguida, o politungstato foi despejado novamente nos tubos com as amostras de solo, onde essa mistura foi submetida à sonificação por 8 minutos e 41 segundos a $60 \%$ de amplitude ( $250 \mathrm{~W}$ ). Terminado esse processo, a mistura foi centrifugada por 30 minutos para a separação da matéria orgânica oclusa. Foi retirado novamente o sobrenadante com auxílio da bomba a vácuo e do filtro e colocados para secar. Por fim os filtros com a matéria orgânica livre e oclusa foram pesados e triturados para a análise do teor de C.

\section{Grau de humificação da matéria orgânica}

As amostras de solo intacto foram secas ao ar, trituradas com um rolo de madeira para passar na malha de $2 \mathrm{~mm}$ e preparadas para análise de Fluorêscencia Induzida por Laser 
(FIL)na Embrapa Instrumentação Agropecuária. A preparação das amostras e a análise de FIL seguiu a metodologia descrita por Milori et al. (2006), na qual alíquotas de aproximadamente $0,5 \mathrm{~g}$ de solo, após serem moídas e passadas em malha de 250 th, são compactadas em paletas de $1 \mathrm{~cm}$ de diâmetro e $2 \mathrm{~mm}$ de espessura e colocadas em um tabuleiro próprio para fazer as medições de FIL. O grau de humificação foi estimado a partir da razão entre a área do espectro de emissão e a porcentagem de carbono total, sendo o resultado obtido para o grau de humificação expresso em u.a (unidade de areia) seguindo o método utilizado por Gonzales-Perez et al. (2007).

\section{Fertilidade do solo}

A análise de fertilidade do solo para a área de estudo foi realizada nos laboratórios de solo da Embrapa Amapá. As análises foram feitas para cada tratamento e profundidade separadamente utilizando amostras compostas de três subamostras. As análises químicas e físicas seguiram os procedimentos utilizados por Silva (2009). Onde foram determinados os teores de matéria orgânica $(\mathrm{MO})$, nitrogênio $(N)$, fósforo $(P)$, potássio $(K)$, cálcio $(\mathrm{Ca})$, cálcio + magnésio $(\mathrm{Ca}+\mathrm{Mg})$, alumínio (Al), hidrogênio + alumínio $(\mathrm{H}+\mathrm{Al})$, soma de bases $(\mathrm{SB})$, saturação de bases $(\% \mathrm{~V})$, saturação por alumínio (\%m), capacidade de troca catiônica (CTC) e pH em H2O. Para textura, foram determinados: a porosidade; densidade aparente; e densidade de partículas.

A fim de verificar a normalidade dos valores resultantes do fracionamento granulométrico e densiométrico e da análise de Fluorescência Induzida por Lazer foi aplicado o teste de normalidade de Kolmogorov-Smirnov.

Para os valores de fracionamento e de FIL obtidos a partir das amostras dos tratamentos aplicados na unidade experimental PACUÍ, foi realizada a análise de variância e o teste de Tukey a $5 \%$ de probabilidade, neste caso utilizando todas as repetições dos tratamentos.

\section{RESULTADOS E DISCUSSÃO}

\section{Fracionamento granulométrico e densiométrico}

De forma geral, todos os tratamentos da unidade experimental PACUÍ demonstraram maior acúmulo de carbono total nas profundidades menores (Figura 3). À medida que se aumentou essa profundidade, observou-se uma redução no estoque de carbono total. No tratamento Q1, por exemplo, a média do teor de carbono total obtido foi de $2,66 \% \pm 0,10 \%$, na profundidade de 0 a $5 \mathrm{~cm}$, e para a maior profundidade de coleta a média obtida foi $1,46 \% \pm 0,08 \%$. Os tratamentos $\mathrm{T}$ e $\mathrm{Q}$ apresentaram maior incorporação de carbono total no solo, quando somados todos os valores por profundidades, com $6,49 \% \pm 0,26 \%$ e $6,14 \% \pm 0,08 \%$, respectivamente.

Figura 3. Porcentagem de carbono total para os tratamentos capoeiras triturada (T), queimada primeiro ciclo (Q1) queimada segundo ciclo (Q2) e capoeira natural (C). Letras maiúsculas iguais, comparação entre tratamentos e letras minúsculas iguais, comparação entre profundidades no tratamento.

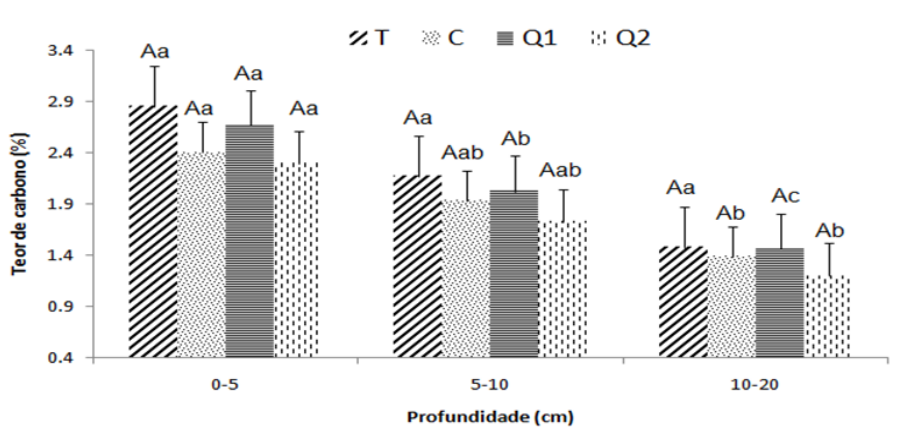

Os tratamentos não apresentaram diferença estatística entre si ( $n=36, p=0,09)$. Porém, os tratamentos $Q 1, Q 2$ e $C$ apresentaram diferença significativa entre as suas profundidades, evidenciando a diminuição no estoque de carbono total com o aumento da profundidade no solo.

O teor de nitrogênio total no solo apresentou baixos percentuais em todos os tratamentos (Figura 4). Observou-se ainda, a redução da quantidade de nitrogênio no solo conforme o aumento na profundidade de coleta das amostras.

Figura 4. Porcentagem de nitrogênio total para os tratamentos capoeiras triturada $(\mathrm{T})$, queimada primeiro ciclo (Q1) queimada segundo ciclo (Q2) e capoeira natural (C). Letras maiúsculas iguais, comparação entre tratamentos e letras minúsculas iguais, comparação entre profundidades no tratamento.

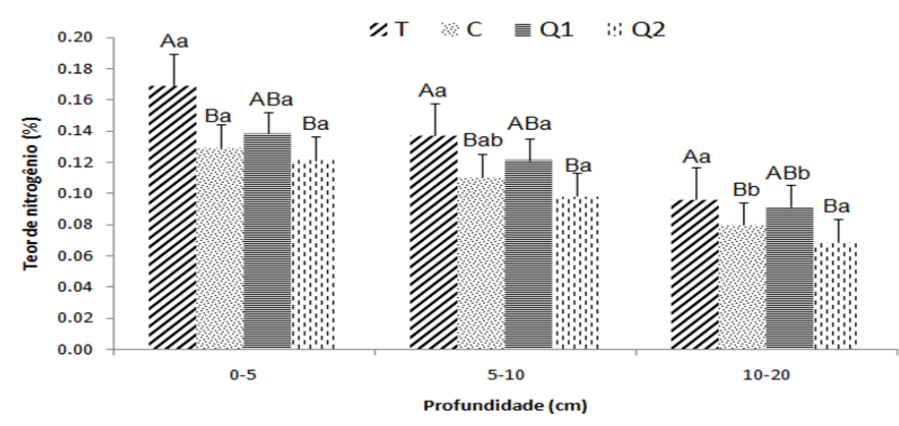

A análise estatística identificou diferenças entre os tratamentos e entre suas profundidades. Essas diferenças ocorreram entre Te $C(n=18, p=0,02)$ e entre T e Q2 $(n=18$, 
$p=0,001)$. O tratamento $Q 1$ apresentou diferença altamente significativa $(p<0,0001)$ entre a menor e a maior profundidade de coleta.

Para matéria orgânica livre, o teor mais elevado foi encontrado nos tratamentos C e Q2, com valor médio de $19,5 \% \pm 0,01 \%$ e $23,6 \% \pm 0,03 \%$ respectivamente na profundidade de 0 a $5 \mathrm{~cm}$ (Figura 5). Estatisticamente ocorreram diferenças significativas entre $T$ e $Q 2(n=18, p=$ 0,042 ). Entretanto, apesar da visível diferença numérica entre Q1 $(8,7 \% \pm 0,06)$ e Q2 $(23,6 \% \pm 0,03 \%)$, não foi constado diferença significativas entre estes tratamentos $(n=18$ e $p=$ $0,05)$. À medida que se aumentou a profundidade de coleta, ocorreram reduções de matéria orgânica livre nos tratamentos T, C e Q2, bem como aumento no tratamento Q1.

Figura 5. Porcentagem de matéria orgânica livre para os tratamentos capoeiras triturada $(\mathrm{T})$, queimada primeiro ciclo (Q1) queimada segundo ciclo (Q2) e capoeira natural (C). Letras maiúsculas iguais, comparação entre tratamentos e letras minúsculas iguais, comparação entre profundidades no tratamento.

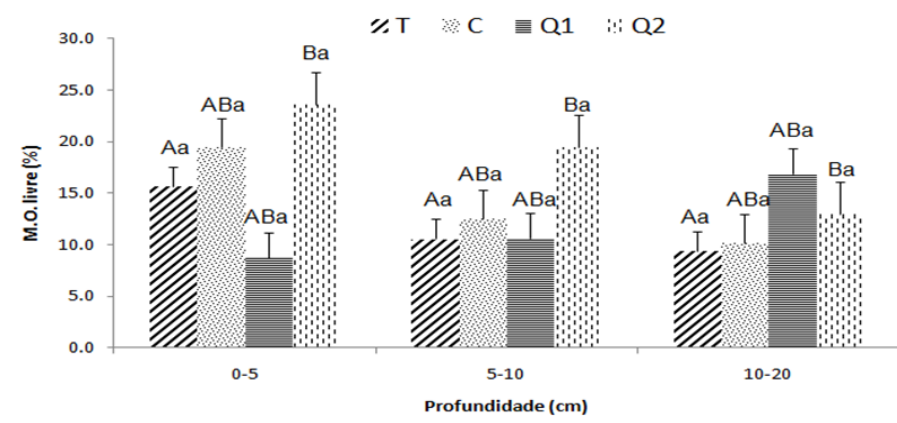

A matéria orgânica oclusa mostrou-se bem distribuída entre as profundidades, sendo $\mathrm{T}$ igual a $23,43 \% \pm 0,15 \%$ na profundidade de 0 a $5 \mathrm{~cm}$ e igual a $16,12 \% \pm 0,21 \%$ na maior profundidade de coleta (10 a $20 \mathrm{~cm}$ ), (Figura 6). Essa semelhança foi confirmada pela análise estatística, que não identificou diferenças significativas entre os tratamentos e entre as profundidades por tratamento $(n=36, p=0,09)$.

Os teores mais elevados de carbono total, em camadas mais superficiais do solo nos tratamentos testados, podem ser atribuídos ao maior aporte de biomassa vegetal acumulada nas camadas superficiais do solo e à atividade microbiana nessas camadas. Em estudo sobre o estoque de carbono orgânico total e nitrogênio total, na agricultura, em solos de cerrado sob sucessão soja milho, Nunes et al. (2011) relataram que o maior estoque de carbono orgânico foi obtido na primeira profundidade de coleta $(0$ a $5 \mathrm{~cm}$ ). Os autores observaram, também, redução no teor de carbono total, conforme o aumento da profundidade de coleta do solo. Essa redução foi atribuída à menor quantidade de resíduos vegetais nas camadas inferiores.
Figura 6. Porcentagem de matéria orgânica oclusa para os tratamentos capoeiras triturada $(\mathrm{T})$, queimada primeiro ciclo (Q1) queimada segundo ciclo (Q2) e capoeira natural (C). Letras maiúsculas iguais, comparação entre tratamentos e letras minúsculas iguais, comparação entre profundidades no tratamento.

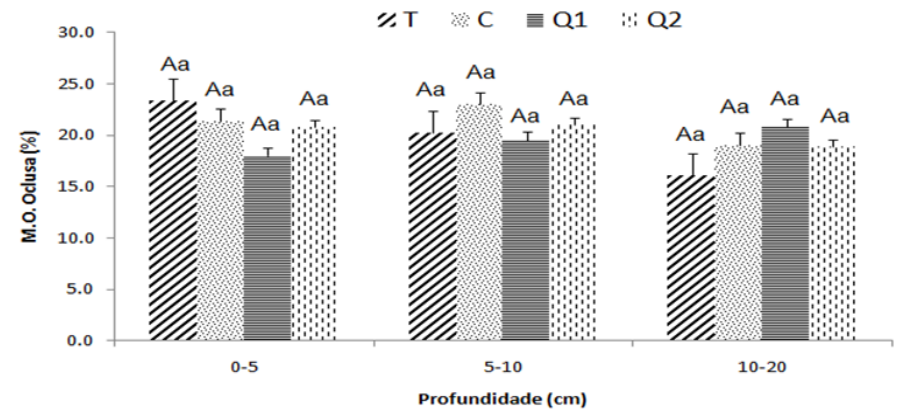

Os resultados obtidos para nitrogênio total, nas primeiras profundidades de coleta nos tratamentos T e C $(0.20 \%$ e $0,15 \%$, respectivamente) podem ser resultantes da decomposição inicial dos resíduos vegetais dispostos sobre o solo. O estoque de nitrogênio total no tratamento Q1 pode ter sofrido acréscimo devido à queima da capoeira, o que proporcionou índices mais elevados que a referência $C$, embora não tenha ocorrido diferença significativa entre esse tratamento e a referência.

Silva (2009) estudou a qualidade do solo, a macro e microfauna em plantios agrícolas sobre a capoeira triturada em solos do cerrado amapaense. No estudo a autora comparou ao cerrado quatro tratamentos: Capoeira triturada; capoeira triturada com adubação alternativa; capoeira triturada com adubação convencional; capoeira triturada queimada. Em todos os tratamentos os teores obtidos para o nitrogênio $(N)$ foram iguais a $0,07 \%$. Esse resultado é próximo dos resultados obtidos na última profundidade de coleta em todos os tratamentos da unidade experimental PACUí. Possivelmente, os baixos teores de $\mathrm{N}$ encontrados por Silva, (2009) foram influenciados pelo pouco tempo de implantação do experimento e pela baixa disponibilidade desse nutriente em solos de cerrado, devido à quantidade de biomassa existente na superfície do solo.

Os resultados encontrados para matéria orgânica livre nos tratamentos T, C e Q2 evidenciam que a formação dessa matéria orgânica está intimamente ligada à deposição da biomassa vegetal sobre o solo. A decomposição dessa biomassa vegetal pela fauna microbiana ocorrentes nas primeiras profundidades de coleta, também pode estar associada à formação dessa matéria orgânica livre.

Em Q1 possivelmente pode ter havido uma queima mais intensa, devido ao tipo de biomassa que foi triturada e depositada sobre o solo, o que pode ter influenciado na 
diferença numérica ente Q1 e Q2. Adicionalmente, essa queima mais intensa pode ter agido sobre a fauna edáfica, reduzindo seu quantitativo com o fogo, o que afetou o potencial de ciclagem e transformação dos resíduos em matéria orgânica livre. Conforme Resk et al. (2008), a fauna edáfica é considerada a principal responsável pela decomposição dos resíduos orgânicos, ciclagem dos nutrientes, fluxo de energia no solo, além de ter como função a transformação da matéria orgânica. De modo que, práticas inadequadas podem afetar diretamente a biologia do solo.

A formação da matéria orgânica oclusa de maneira homogênea em todas as profundidades dos tratamentos em PACUÍ pode estar relacionada, à tipologia florestal da área e a deposição dos resíduos vegetais sobre o solo. Em PACUÍ a tipologia florestal proporcionou uma determinada quantidade de biomassa. Isso pode ter implicado em maior quantidade de matéria orgânica a ser decomposta. Correia e Andrade (2008) relatam que a decomposição da biomassa depositada sobre o solo, relaciona-se a ação decompositora dos microrganismos do solo. Quanto maior a quantidade de biomassa depositada e menor a velocidade de decomposição mais tempo a matéria orgânica permanece no solo que favorece a formação de matéria orgânica oclusa. Os tratamentos T, Q1, e Q2 também podem ter influenciado na formação da matéria orgânica oclusa, causando efeitos que, proporcionaram a retenção da matéria orgânica em formas menos acessíveis a microfauna do solo.

\section{Fluorescência induzida por laser em São Joaquim do Pacuí}

Nos tratamentos da unidade experimental de PACUÍ o grau de humificação aumentou conforme o aumento na profundidade de coleta das amostras (Figura 7). $\mathrm{Na}$ profundidade de 10 a $20 \mathrm{~cm}$, os tratamentos $\mathrm{T}$ e Q2 apresentaram maior humificação da matéria orgânica.

Em todos os tratamentos observou-se um grau de humificação similar, o que foi comprovado na análise estatística, que não identificou diferença significativa entre os tratamentos. Contudo, para os tratamentos $\mathrm{T}$ e Q2 foi observada diferença significativa ( $n=18$ e $p<0,0001$ ) entre as profundidades de 0 a $5 \mathrm{~cm}$ e 10 a $20 \mathrm{~cm}$. Sendo que os tratamentos $\mathrm{T}(213.93 \% \pm 0,12 \%)$ e $\mathrm{Q} 1(222,23 \% \pm 0,26 \%)$ obtiveram maior humificação da matéria orgânica do solo.

Os tratamentos não ocasionaram grande influência na humificação da matéria orgânica do solo na profundidade de 0 a $5 \mathrm{~cm}$. À medida que se aumentou a profundidade de coleta ocorreu aumento no grau de humificação da matéria orgânica em todos os tratamentos. Porém, apesar de Q1 não se diferir dos demais tratamentos, a queima dos resíduos triturados provocou a diferença ao longo das profundidades do solo, reduzindo a humificação nesse tratamento. Isso pode ter ocorrido devido às perdas por volatilização de boa parte dos nutrientes contidos na vegetação. A partir do segundo ciclo de cultivo (Q2), a implantação das culturas pode ter reestabelecido a humificação perdida inicialmente.

Figura 7. Grau de humificação da matéria orgânica para os tratamentos capoeiras triturada $(\mathrm{T})$, queimada primeiro ciclo (Q1) queimada segundo ciclo (Q2) e capoeira natural (C). Letras maiúsculas iguais, comparação entre tratamentos e letras minúsculas iguais, comparação entre profundidades no tratamento.

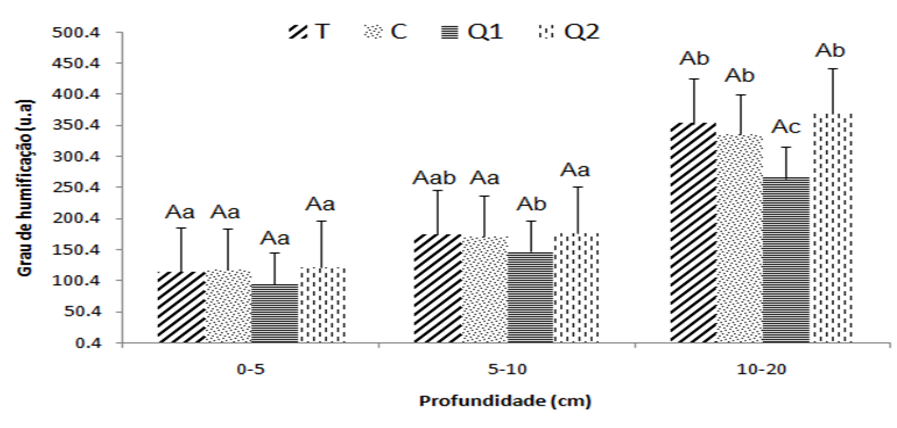

Favoretto et al. (2008) estudaram a humificação em solos sob cultivos com plantio direto, plantio convencional e preparo mínimo através da Fluorescência Induzida por Laser. Os autores também encontram valores crescentes de humificação, à medida que ocorreu o aumento na profundidade de coleta. Esses resultados foram atribuídos ao aporte de biomassa fresca depositados nas camadas mais superficiais do solo. À medida que se aumenta a profundidade de coleta, maior pode ser a decomposição e a humificação da matéria orgânica.

\section{Fertilidade do solo}

Os tratamentos para a unidade de PACUÍ não se diferiram com relação ao teor de argila com valores variando entre 111 a $261 \mathrm{~g} \mathrm{~kg}^{-1}$ (Tabela 2). Para a areia grossa os teores mais elevados foram quantificados nos tratamentos C e Q1 (489 e $497 \mathrm{~g} \mathrm{~kg}^{-1}$, respectivamente). Os teores de areia fina foram menores no tratamento $\mathrm{T}\left(153 \mathrm{~g} \mathrm{~kg}^{-1}\right)$ que também obteve maiores índices para o silte $\left(263 \mathrm{~g} \mathrm{~kg}^{-1}\right)$.

Com relação às características químicas para a fertilidade do solo, observa-se que os valores de $\mathrm{pH}$ variaram entre 4,7 e 5,3 (Tabela 3). Os teores de matéria orgânica do solo foram iguais nos tratamentos C, Q1 e Q2. Em T, a menor profundidade demonstrou maior acúmulo de matéria orgânica $\left(51,3 \mathrm{gkg}^{-1}\right)$. 
Tabela 2. Análise granulométrica para os elementos texturais do solo na unidade experimental do PACUÍ, para os tratamentos capoeiras triturada (T), queimada primeira ciclo (Q1), queimada segundo ciclo (Q2) e capoeira natural (C). Letras maiúsculas iguais, comparação entre tratamentos e letras minúsculas iguais, comparação entre profundidades no tratamento.

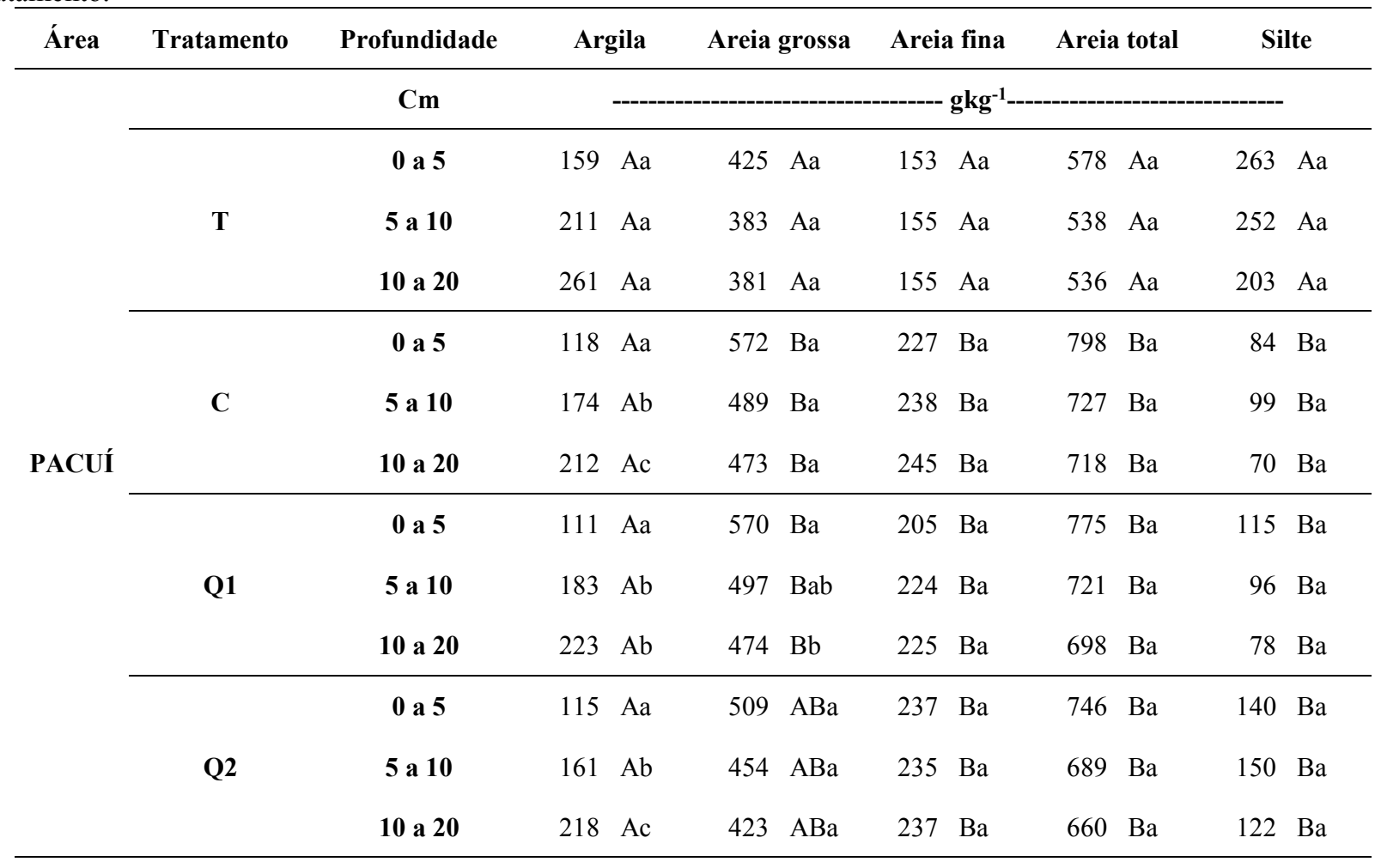

Os valores para o fosfato (P) foram maiores nas profundidades de 0 a $5 \mathrm{~cm}$ dos tratamentos $\mathrm{T}\left(12 \mathrm{mg} \mathrm{m}^{-3}\right) \mathrm{e}$ Q1 $\left(9 \mathrm{mg} \mathrm{m}^{-3}\right)$. Nos demais tratamentos, os valores não ultrapassaram $3 \mathrm{mg} \mathrm{m}^{-3}$, no entanto, não foi observada diferença significativa nos tratamentos e nas profundidades.

A CTC foi maior nos tratamentos T e Q1, com $11,8 \mathrm{cmdcm}$ ${ }^{3}$ para os dois tratamentos.

Os resultados observados para a fração de argila do solo nos tratamentos de PACUÍ indicam baixa capacidade do solo em reter matéria orgânica. Com relação aos atributos químicos, o pH não sofreu grandes alterações. $\mathrm{O}$ tratamento $\mathrm{Q} 2$, que se diferenciou de $\mathrm{T}$, diminuiu o nível de acidez no solo. O tratamento T aumentou a CTC, assim como o tratamento Q1. Porém, a partir do segundo ciclo (Q2) observa-se que a CTC é reduzida a níveis menores que observado para a referência, indicando que sistemas com o uso do fogo no preparo do solo podem ocasionar baixa CTC em cultivos em longa duração.

\section{CONCLUSÕES}

$\mathrm{Na}$ unidade experimental do PACUÍ, os sistemas de manejo com trituração da capoeira $(T)$ e queima da capoeira no primeiro ciclo de cultura (Q1) indicaram maior incorporação de carbono total no solo.

Esses mesmos sistemas apresentaram maior grau de humificação da matéria orgânica do solo para $T$ e Q1, confirmando o que foi observado em relação ao teor de carbono total do solo.

Os sistemas de manejo do solo com capoeira triturada (T), e queima da capoeira no segundo ciclo (Q2) favoreceram a fertilidade do solo, proporcionando condições favoráveis ao acúmulo de matéria orgânica na unidade experimental do PACUÍ. Confirmando as observações relacionadas ao o teor de carbono total nos tratamentos.

Entretanto, deve-se salientar a necessidade de continuação desses estudos para confirmar os resultados obtidos para o campo experimental, uma vez que pode ter sido influenciado pela duração do experimento, a qual foi de apenas dois anos.

\section{REFERÊNCIAS}

BERVALD, C. M. P. Tecnologia mecanizada em preparo de área sem Queima no nordeste paraense. 2005. 73 p. Dissertação (Mestrado em Mecanização Agrícola) Universidade Federal de Santa Maria, Santa Maria, 2005. BERVALD, C. M. P; KATO. O. R.; REICHERT, J. M.; REINERT, D. J. Agregados A Seco compressibilidade de latossolo amarelo 
submetido a preparos tradicionais e alternativo na amazônia oriental. [s.I.: s.n.] [2003?]. 4p.

BOSS, R. L.. Variações espaciais e temporais em comununidades de aves de uma savana amazônica no estado do Amapá Macapá - AP 2009, 154p. Dissertação (Mestrado em solos)- Universidade Federal do Amapá, Macapá, 2009.

CARPENEDO, V.; MIELNICZUK, J. Estado de agregação e qualidade de latossolos roxos submetidos a diferentes sistemas de manejo. Revista brasileira de ciências do solo, n14. 99-105 p. 1990.

CIRNE, M. B.; SOUZA, A. G. S. M. POUSIO: o que é e quais são os seus possíveis reflexos nas questões ambientais. Veredas do Direito, Belo Horizonte. v.11, n.21, p.75-106 Janeiro/junho de 2014.

CORRÊA; J. C. Efeito de sistemas de cultivo na estabilidade de agregados de um Latossolo Vermelho-Amarelo em Querência, MT. Pesquisa agropecuária brasileira, Brasília, v. 37, n. 2, p. 203-209, fev. 2002.

CORRÊA, F. E. M.; ANDRADE, A. G. Formação de serapilheira e ciclagem de nutrientes. GUERRA, J.M.G,;SANTOS,G. A.; SILVA, S. L.;CAMARGO, F.A.O. Fundamentos da matéria orgânica do solo, ecossitemas tropicais e subtropicais. Porto Alegre, Metrópole, 2008. p. 137-158.

CHRISTENSEN B.T. Physical fractionation of soil and structural and functional complexity in organic matter turnover. Journal, European of Soil Science, 345-353p. 2001. Supplementum 52.

CONCEIÇÃO, C. P.; BOENI, M.; DIECKOW, J.; BAYER.C.; MARTINNETO, L.; MIELNICZUK, J. Eficiência do politungstato de sódio no fracionamento densiométrico da matéria orgânica do solo. Revista brasileira de ciências do solo, V 31, 9 p. 2007.

CONCEIÇÃO, C. P.; BOENI, M.; DIECKOW, J.; BAYER.C.; MIELNICZUK, J. Fracionamento densiométrico com politungstato de sódio no estudo da matéria orgânica do solo. Revista brasileira de ciências do solo, V 32, 9 p. 2008.

FAVORETO, C. M. Caracterização da matéria orgânica humificada de um latossolo Vermelho distrófico através da espectroscopia de fluorescência induzida por laser 2007. 99 p. Dissertação (Mestrado em Química Aplicada)Universidade Federal de Ponta Grossa, Santa Maria, 2007.

FAVORETTO, C. M.; GONÇALVES, D.; Milori, D. M. B. P.; Rosa, J. A.; SAAB, S. C.; LEITE, W.C.; BRINATTI, A. M. Determinação da humificação da matéria orgânica de um latossolo e de suas frações organo-minerais. Química Nova, Vol. 31, n 8, 1994-1996 p. 2008.

FEARNSIDE P. M. Amazonian deforestation and global warming: carbon stocks in vegetation replacing Brazil's Amazon forest. Forest ecology and management, v. 80, p. 21-34. 1996
FEARNSIDE, P. M. Uso da terra na Amazônia e as mudanças climáticas globais. SEB, indd 10-2 p. 2007.

FONTANA, A.; ANJOS, L. H. C; SALLÉS, J. M.; PEREIRA, M. G.; ROSSIELLO, R. O. P. Carbono orgânico e fracionamento químico da matéria orgânica em solos da Sierra de Ánimas. Uruguai, V.12, n.1, p. 36 - 43, 2005.

GONZALES-PERES M.; MILORI D.M.B.P.; COLNAGO L.A.; MARTIN-NETO L.; MELO W.J. A laser-induced fluorescence spectroscopic study of organic matter in a Brazilian Oxisol under different tillage systems. [S.I.], Geoderma, 2007. 138: 20-24p.

GUERRA, J.M.G,;SANTOS,G. A.; SILVA, S. L.;CAMARGO, F.A.O. Macromeculas e substâncias húmicas.In:. Fundamentos da matéria orgânica do solo, ecossistemas tropicais e subtropicais. Porto Alegre, Metrópole, 2008. p. 19-20.

INSTITUTO DE PESQUISAS CIENTÍFICAS E TECNOLOGICAS ESTADO DO AMAPÁ (Macapá). Macrodiagnóstico do estado do Amapá, primeira aproximação do ZEE. Amapá, 2008, 90-139 p.

JÚNIOR, N. N. P.; MURRIETA, R. S. S.; ADAMS, C. A agricultura de corte e queima: um sistema em transformação. Emílio Goeldi. Ciências Humanas, Belém, v. 3, n. 2, p. 153-174, maio - ago. 2008.

KATO, M.S.A.; KATO O.R., DENICH, M.; VLEK, P.L.G. Fire-free alternatives to slash-and-burn for shifting cultivation in the eastern Amazon region: the role of fertilizers. Field Crops Research, v.62, p. 225- 237.1999.

LAMPLACHT, H. Silvicultura en los trópicos. Instituto de silvicultura da Universidade de La Göttingen, 1990, p.107112.

MACKENSEN, J.; HGLSCHER, D.; KLINGE, R.; FGLSTER, H. Nutrient transfer to the atmosphere by burning of debris in eastern Amazonia. Forest Ecology and Management. V. 86, p.122-128. 1996.

MILORI D.M.B.P.; GALETI H.V.A.; MARTIN-NETO L.; DIECKOW J.; GONZÁLEZ-PÉREZ M.; BAYER C.; SALTON J. Organic Matter Study of Whole Soil Samples Using Laser-Induced Fluorescence Spectroscopy. Journal, Soil Science Society of American. p.57-63. 2005 Suplemento 70.

MINISTÉRIO DAS MINAS E ENERGIA DEPARTAMENTO NACIONAL DA PRODUÇÃO MINERAL PROJETO RADAM (Macapá). Levantamento de recursos naturais. Amapá, 9174, 462p.

NUNES, R. S.; LOPES, A. A. C.; MARTINHÃO, D. SOUSA, G.; MENDES, I. C. Sistemas de manejo e os estoques de carbono e nitrogênio em latossolo de cerrado com a sucessão soja-milho. Revista Brasileira. Ciências do Solo, 35:1407-1419, 2011.

NUNES, R. S.; SOUSA, D. M. G.; GOEDERT, W. J.; VIVALDI, L. J. DISTRIBUIÇÃO de fósforo no solo em razão do sistema de cultivo e manejo da adubação fosfatada. Revista Brasileira. 
Ciências do Solo, 35: 1407-1419, 35:877-888, 2011.

PAINEL INTERGOVERNAMENTAL DAS MUDANÇAS

CLIMÁTICAS (Genebra, Suíça) 2007: A base das Ciências físicas. Genebra, Suíça 2007. 940 p.

ROSCOE, R.; MACHADO, P. L. O. A. Fracionamento físico em estudo de matéria orgânica. Dourados, MS, Embrapa Agropecuária Oeste, 2002. 86 P.

ROSCOE. R.; BOODEY, R. M.; SALTON J. C. Sistemas de manejo e matéria orgânica do solo In: ROSCOE. R.; F. M. MERCANTE.; SALTON J. C. Dinâmica da matéria orgânica do solo em sistemas conservacionistas, modelagem matemática e métodos auxiliares. Dourados, MS, Empresa brasileira pesquisa agropecuária, 2006. 324 p.

SALTON, J. C.; MIELNICZUK, J.; BAYER, C.; FABRÍCIO, A. C.; MACEDO, M. C.Teor e dinâmica do carbono no solo em sistemas de integração lavoura pecuaria Pesquisa Agropecuária brasileira, Brasília, v.46, n.10, p.1349-1356, out. 201.

SAMPAIO, C; KATO, O. R.; NASCIMENTO-E-SILVA, D. A. Sistema de corte e trituração da capoeira sem queima como alternativa de uso da terra, rumo à sustentabilidade florestal no nordeste paraense. Revista de Gestão Social e Ambiental. v. 2, n. 1, Jan. - Abr, 2008, p.41-53.

SANTANA, D. R. M. M. Estudo de casos na microrregião bragantina do nordeste paraense - PA: Alternativas as práticas tradicionais da agricultura familiar. 2008. 66 p. Monografia (Engenharia Florestal) Universidade Federal Rural do Rio de Janeiro, Seropédica, 2008.

SCHMITZ, H. Transição da agricultura itinerante na Amazônia para novos sistemas Resumos do II Congresso Brasileiro de Agroecologia. Revista brasileira Agroecologia, v.2, n.1, p 46 a 49, fev. 2007.

SILVA, D. M. M. H. Macrofauna edáfica, biomassa microbiana e Qualidade do solo em área cultivada no cerrado Amapaense com e sem uso do fogo e adubação Alternativa. 2009. 80 p. Dissertação (Mestrado em solos)Universidade Federal do Amapá, Macapá, 2009.

Submissão: 18/09/2019

Aprovado para publicação: 30/10/2019 


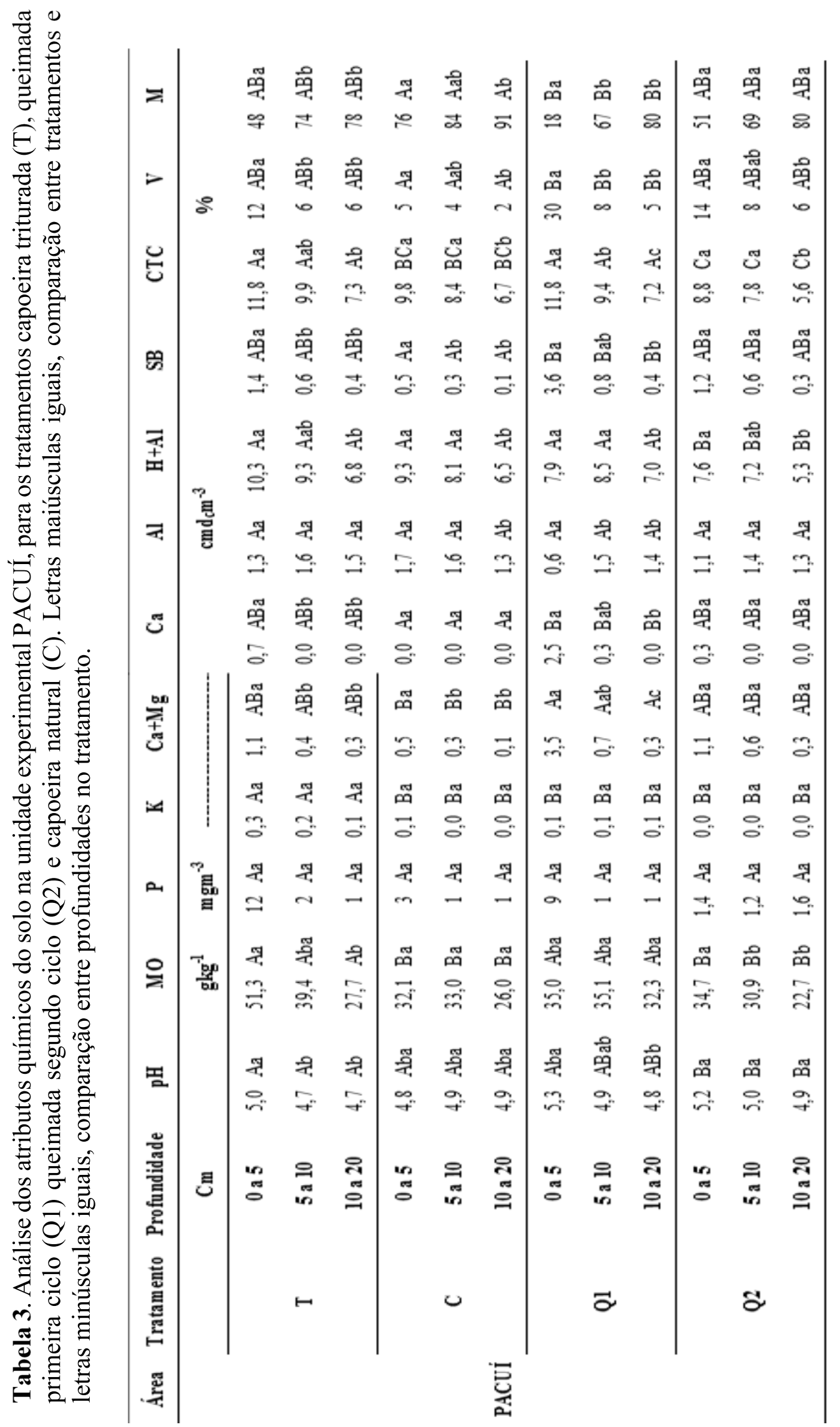

